\title{
São as Tarefas de Nomeação Seriada Rápida Medidas do Processamento Fonológico?
}

\section{Are Rapid Automatized Naming Tasks Measures of Phonological Processing?}

\author{
Cláudia Nascimento Guaraldo Justi ${ }^{*}, a$, Antonio Roazzi ${ }^{b}$ \& Francis Ricardo dos Reis Justi ${ }^{a}$ \\ ${ }^{a}$ Universidade Federal de Juiz de Fora, Juiz de Fora, Minas Gerais, Brasil \\ $\&^{b}$ Universidade Federal de Pernambuco, Recife, Pernambuco, Brasil
}

\begin{abstract}
Resumo
O presente estudo avaliou, por meio da modelagem de equação estrutural, a estrutura fatorial subjacente às medidas de consciência fonológica, memória de trabalho fonológica e nomeação seriada rápida, tendo em vista o conflito na literatura no que se refere à natureza das tarefas de nomeação seriada rápida. Foram comparados modelos compatíveis com a proposta de que as tarefas de consciência fonológica, memória fonológica e nomeação seriada rápida avaliam o processamento fonológico e modelos compatíveis com a proposta de que tarefas de nomeação seriada rápida não avaliam o processamento fonológico. Os resultados desse estudo oferecem suporte à hipótese de que a nomeação seriada rápida não é uma medida do processamento fonológico.

Palavras-chave: Processamento fonológico, nomeação seriada rápida, consciência fonológica, memória de trabalho fonológica, leitura.

Abstract

Considering a conflict in literature regarding the nature of rapid automatized naming tasks, this study evaluated the factor structure underlying the measures of phonological awareness, phonological working memory and rapid automatized naming tasks using the method of structural equation modeling (SEM). Models compatible with the proposal that the tasks of phonological awareness, phonological working memory and rapid automatized naming assess phonological processing, and models compatible with the proposal that rapid automatized naming tasks do not assess phonological processing were compared. The results of this study support the hypothesis that rapid automatized naming tasks are not measures of phonological processing.

Keywords: Phonological processing, rapid automatized naming, phonological awareness, phonological working memory, reading.
\end{abstract}

Consciência fonológica, memória de trabalho fonológica e nomeação seriada rápida estão entre as variáveis preditivas da leitura e da escrita mais estudadas (Bryant \& Bradley, 1987; de Jong \& van der Leij, 1999; Norton \& Wolf, 2012; Wagner \& Torgesen, 1987; Wolf \& Bowers, 1999). A consciência fonológica refere-se à habilidade de refletir sobre os sons que compõem a fala (Bryant \& Bradley, 1987), sendo, dessa forma, um tipo de habilidade metalinguística. A memória de trabalho fonológica relaciona-se ao funcionamento da 'alça fonológica' do modelo de memória de trabalho de Baddeley (2000) e

\footnotetext{
"Endereço para correspondência: Instituto de Ciências Humanas, Universidade Federal de Juiz de Fora, Campus Universitário, Juiz de Fora, MG, Brasil, 36036-330. E-mail: claudia.ngjusti@gmail.com Os autores agradecem ao professor Jorge Artur Peçanha de Miranda Coelho (Universidade Federal de Alagoas) por seus valiosos comentários e auxílio no que se refere ao uso da modelagem de equação estrutural.

Os autores agradecem, também, ao Conselho Nacional de Desenvolvimento Científico e Tecnológico (CNPq) por financiar suas atividades.

Esse estudo é parte integrante da tese de doutorado da primeira autora.
}

pode ser compreendida a partir da interação entre um armazenamento fonológico passivo que mantém a informação disponível por um curto período de tempo e um processo articulatório ativo que permite a manutenção da informação fonológica por um período indefinido de tempo graças a sua constante atualização via subvocalização ou vocalização. A nomeação seriada rápida refere-se à habilidade de nomear estímulos visuais, apresentados em série em um cartão, o mais rápida e corretamente quanto possível, sendo a natureza dessa tarefa foco de um considerável debate na literatura (Wagner \& Torgesen, 1987; Wolf \& Bowers, 1999).

Uma forma de integrar as pesquisas realizadas acerca dessas variáveis foi proposta por Wagner e Torgesen (1987; Wagner, Torgesen, Laughon, Simmons, \& Rashotte, 1993). De acordo com esses pesquisadores, essas três variáveis relacionar-se-iam a diferentes componentes do processamento fonológico, sendo o termo 'processamento fonológico' definido pelos autores como ". . . o uso da informação fonológica (os sons da própria língua) no processamento da linguagem oral e escrita" (Wagner \& Torgesen, 1987, p. 192). 
Enquanto a consciência fonológica ajudaria na compreensão do princípio alfabético, a memória de trabalho fonológica seria importante para a leitura porque capacitaria o leitor iniciante a manter uma representação precisa dos fonemas associados com letras ou grupos de letras, enquanto ele devotaria uma grande quantidade de recursos para processos de decodificação e compreensão (Wagner et al., 1993; Wagner et al., 1997). Já a nomeação seriada rápida, por envolver a recuperação e a pronúncia do nome de estímulos visuais, indexaria a eficiência com que códigos fonológicos são recuperados da memória de longo prazo. Para Wagner et al. (1993) “. . . a eficiência com que as crianças são capazes de recuperar códigos fonológicos associados com fonemas individuais, palavras segmentadas ou palavras inteiras deve influenciar o grau em que a informação fonológica é aproveitada na decodificação" (p. 84). Assim sendo, para Wagner e Torgesen (1987), consciência fonológica, codificação fonológica na memória de trabalho e recuperação de códigos fonológicos da memória de longo prazo seriam três componentes do processamento fonológico, e um problema no processamento fonológico seria a principal causa de dificuldade no desenvolvimento da leitura.

A proposta de Wagner e Torgesen (1987) se tornou bastante influente e, até hoje, a correlação entre o desempenho em tarefas de nomeação seriada rápida e o desempenho em tarefas de leitura tem sido interpretada, frequentemente, como mais uma evidência acerca da relação existente entre o processamento fonológico e a leitura (Taibah \& Haynes, 2011; Vandewalle, Boets, Ghesquière, \& Zink, 2012). Uma implicação prática diretamente relacionada a essa proposta é que as crianças que apresentam, subjacente à dificuldade de leitura, uma dificuldade com a nomeação seriada rápida, são submetidas a intervenções que enfatizam, primordialmente, a estimulação do processamento fonológico, o que nem sempre é eficaz (Lovett, Steinbach, $\&$ Frijters, 2000). Esse fato se torna ainda mais relevante ao se considerar que existem controvérsias quanto à adequação de se conceber as tarefas de nomeação seriada rápida como medidas do processamento fonológico (Wolf \& Bowers, 1999). De acordo com Wolf e Bowers (1999), crianças com dificuldade de leitura que apresentam uma lenta nomeação seriada não se beneficiariam de um programa de intervenção que tem como foco, exclusivo, a estimulação do processamento fonológico, uma vez que tarefas de nomeação seriada rápida não seriam um índice do processamento fonológico. Destarte, aqui se tem um embate teórico com importantes consequências para a prática psicológica no que diz respeito ao diagnóstico e à remediação de dificuldades de leitura.

Em uma tarefa de nomeação seriada rápida o participante nomeia, tão rápida e corretamente quanto possível, um conjunto de cinquenta estímulos visuais, todos de alta frequência de ocorrência, dispostos em série em um cartão. Os estímulos visuais a serem nomeados podem ser: quadrados coloridos; números; objetos desenhados; e, letras, dependendo da tarefa (Denckla \& Rudel, 1976).
De acordo com Wolf e Bowers (1999), apesar do desempenho nas tarefas de nomeação seriada rápida envolver a recuperação de códigos fonológicos da memória de longo prazo, a ênfase sobre a velocidade de processamento e a exigência de que vários outros processos cognitivos funcionem de forma sincronizada e que sejam integrados, impõem a necessidade de uma categorização própria para as tarefas de nomeação seriada rápida. Segundo as pesquisadoras, essas tarefas compreendem um conjunto de processos de natureza perceptual (necessários para o reconhecimento do padrão visual, ou seja, para a discriminação e a identificação dos estímulos visuais), de natureza lexical (necessários para o acesso e a recuperação de informações ortográficas, fonológicas e semânticas) e de natureza motora (necessários para a articulação da resposta). Wolf e Bowers (1999) ressaltam a precisão, a automaticidade e a sincronia em cada um e entre esses vários processos, bem como a integração eficiente dos mesmos, como fatores críticos para o bom desempenho nas tarefas de nomeação seriada rápida. Nesse sentido, a nomeação seriada rápida seria um índice da eficiência com que múltiplos processos cognitivos são automatizados, sincronizados e integrados, sendo a sincronia entre os processos um aspecto fundamental para a integração dos mesmos. Wolf e Bowers (1999) argumentam que, embora os processos lexicais envolvam a recuperação de códigos fonológicos da memória de longo prazo, tal envolvimento não justifica a classificação das tarefas de nomeação seriada rápida como uma medida do processamento fonológico, afinal, tarefas de fluência semântica e tarefas de vocabulário expressivo também pressupõem a recuperação de representações fonológicas da memória de longo prazo e, nem por isso, são classificadas como medidas do processamento fonológico. Dessa forma, pode-se dizer que a principal discordância entre Wagner e Torgesen (1987) e Wolf e Bowers (1999) é quanto à adequação de se categorizar as tarefas de nomeação seriada rápida como indexando, primordialmente, o processamento fonológico.

Tendo como objetivo principal examinar a relação entre os três componentes do processamento fonológico, conforme proposto por Wagner e Torgesen (1987), Wagner et al. (1993) desenvolveram um estudo no qual várias tarefas que, segundo os autores, avaliam cada um dos três componentes do processamento fonológico, foram administradas e diferentes modelos de mensuração foram avaliados por meio da modelagem de equação estrutural. Quanto aos resultados encontrados, de forma consistente com os resultados dos demais estudos realizados por esses autores (Wagner et al., 1987; Wagner, Torgesen, \& Rashotte, 1994; Wagner et al., 1997), as medidas de nomeação seriada rápida ficaram em um fator separado nos modelos com melhores índices de ajuste. Assim sendo, Torgesen, Wagner, Rashotte, Burgess e Hecht (1997) e Wagner et al. (1993) têm destacado apenas a correlação existente entre as medidas de nomeação seriada rápida $\mathrm{e}$ as medidas de consciência fonológica e de memória de trabalho fonológica, bem como a validade de face, como argumentos que justificam a inclusão das tarefas de no- 
meação seriada rápida como parte do que eles chamaram de 'família fonológica'.

Considerando a proposta de Wagner e Torgesen (1987) e a proposta alternativa de Wolf e Bowers (1999), Powell, Stainthorp, Stuart, Garwood e Quinlan (2007) desenvolveram um estudo com o objetivo de investigar qual dessas duas hipóteses teóricas era mais consistente com os dados utilizando, para isso, também, a modelagem de equação estrutural. Nessa pesquisa, três modelos de mensuração foram avaliados por meio de análises fatoriais confirmatórias. Os resultados evidenciaram que tanto o modelo de três fatores ('consciência fonológica'; 'memória fonológica' e 'nomeação seriada rápida') como o modelo de dois fatores ('processamento fonológico' - indicado pelas medidas de consciência fonológica e de memória fonológica - e 'nomeação seriada rápida' - indicado pelas medidas de nomeação seriada rápida) foram consistentes com os dados. O modelo que incluiu apenas um único fator ("processamento fonológico') como sendo responsável pelas variações em todas as medidas de consciência fonológica, de memória fonológica e de nomeação seriada rápida não foi consistente com os dados. De acordo com Powell et al. (2007), os resultados de seu estudo são consistentes com a proposta de Wolf e Bowers (1999), pois evidenciam que os processos subjacentes à nomeação seriada rápida são, pelo menos parcialmente, independentes daqueles processos que subjazem, de uma forma mais geral, as tarefas de consciência fonológica e de memória fonológica.

Outros estudos investigaram a estrutura fatorial subjacente às medidas de consciência fonológica, de memória fonológica e de nomeação seriada rápida por meio da modelagem de equação estrutural e os resultados encontrados foram semelhantes aos de Powell et al. (2007), seja ao indicarem o modelo de três fatores - 'consciência fonológica', 'memória fonológica' e 'nomeação seriada rápida' - como o mais consistente com os dados (Anthony, Williams, McDonald, \& Francis, 2007; de Jong \& van der Leij, 1999; Wagner et al., 1993), seja ao indicarem o modelo de dois fatores - 'processamento fonológico' e 'nomeação seriada rápida' - como sendo o modelo mais consistente com os dados (Lonigan et al., 2009; Wagner et al., 1987). No entanto, com exceção de Powell et al. (2007), os demais pesquisadores interpretam os resultados de seus estudos como sendo consistentes com a hipótese de que as tarefas de consciência fonológica, de memória fonológica e de nomeação seriada rápida avaliam o processamento fonológico, uma vez que os fatores se correlacionam. Assim sendo, é importante que sejam tecidas algumas considerações acerca desses estudos.

Uma primeira questão refere-se ao argumento de que os modelos de dois e de três fatores são consistentes com a hipótese de Wagner e Torgesen (1987) porque os fatores se correlacionam. Esse argumento, apesar de à primeira vista parecer razoável, enfrenta sérias dificuldades, a não ser que se argumente, também, que tarefas utilizadas para avaliar a inteligência avaliam o processamento fonológico. Afinal, nos resultados relatados por Anthony et al. (2007), de Jong e van der Leij (1999) e Wagner et al. (1993), o fator 'consciência fonológica' apresentou uma correlação mais forte com o fator 'inteligência' do que com o fator 'nomeação seriada rápida'.

Outra questão importante é que, apesar do modelo de um único fator representar a hipótese forte de que o processamento fonológico é o responsável pelas variações nas medidas de consciência fonológica, de memória fonológica e de nomeação seriada rápida, esse modelo não parece representar adequadamente a proposta de que essas tarefas avaliam tipos de processamento fonológico diferentes, que é, exatamente, a proposta de Wagner e Torgesen (1987). Talvez, o modelo que melhor avalie essa hipótese seja um modelo que inclua um fator de segunda ordem ('processamento fonológico') como sendo responsável por variações em fatores de primeira ordem ('consciência fonológica', 'memória fonológica' e 'nomeação seriada rápida'). No entanto, até o presente momento, parece que esse modelo ainda não foi avaliado e, além disso, nenhum dos outros modelos foi avaliado em falantes da língua portuguesa.

Considerando-se que há duas perspectivas conflitantes na literatura a respeito da natureza "fonológica" das tarefas de nomeação seriada rápida (Wagner \& Torgesen, 1987; Wolf \& Bowers, 1999), o presente estudo teve como objetivo comparar, por meio da modelagem de equação estrutural, modelos de mensuração derivados dessas duas propostas teóricas. Nesse estudo, a hipótese de Wagner e Torgesen (1987) foi representada por dois modelos: (a) Modelo 1 - modelo no qual as variáveis observadas (escores nas tarefas de consciência fonológica, de memória fonológica e de nomeação seriada rápida) são indicadoras de uma mesma variável latente, denominada 'processamento fonológico'; e, (b) Modelo 2 - modelo no qual o 'processamento fonológico' é um fator de segunda ordem e as variáveis latentes 'consciência fonológica', 'memória fonológica' e 'nomeação seriada rápida' são fatores de primeira ordem ${ }^{1}$. Já a proposta de Wolf e Bowers (1999) foi representada, também, por dois modelos: (a) Modelo 3 - modelo em que três variáveis latentes ('consciência fonológica', 'memória fonológica' e 'nomeação seriada rápida') se correlacionam, mas no qual não há um fator de segunda ordem representando uma fonte comum de variação a essas três variáveis; e, (b) Modelo 4 - modelo em que há duas variáveis latentes ('processamento fonológico' - consciência fonológica e memória fonológica - e 'nomeação seriada rápida') que se correlacionam. A representação gráfica desses modelos encontra-se na seção 'Resultados'.

Ao se avaliar esses quatro modelos, se os Modelos 1 ou 2 se mostrarem consistentes com os dados, sendo 
os caminhos que ligam as variáveis latentes às variáveis observadas e os caminhos que ligam o fator de segunda ordem aos fatores de primeira ordem todos significativos, então, pode-se dizer que a proposta de Wagner e Torgesen (1987) foi corroborada pelo presente estudo. Afinal, essa evidência favorece a hipótese de que variações na nomeação seriada rápida advêm, primordialmente, de variações no processamento fonológico, sendo as medidas de nomeação seriada rápida, portanto, adequadas para avaliar o processamento fonológico. Caso contrário, pode-se dizer que a hipótese teórica de Wagner e Torgesen não recebeu suporte empírico no presente estudo. No entanto, se os Modelos 3 ou 4 forem consistentes com os dados e o fator 'nomeação seriada rápida' não se correlacionar fortemente com os demais fatores, então é a hipótese de Wolf e Bowers (1999) que será corroborada. Afinal, esses modelos representam a ideia de que as tarefas de nomeação seriada rápida avaliam, primordialmente, processos diferentes dos avaliados pelas tarefas de consciência fonológica e de memória de trabalho fonológica (daí a nomeação seriada rápida ser representada por um fator separado dos demais fatores e as correlações entre o fator 'nomeação seriada rápida' e os demais fatores não poder ser forte). Caso os resultados sigam em direção contrária, pode-se dizer que a hipótese de Wolf e Bowers não recebeu suporte empírico no presente estudo ${ }^{2}$.

\section{Método}

\section{Participantes}

A amostra foi constituída por 94 crianças matriculadas em três escolas particulares localizadas na cidade do Recife e que se encontravam no quarto ano do ensino fundamental. A idade das crianças variava de oito anos e meio até dez anos e dez meses, sendo a média igual a nove anos e três meses (desvio padrão de 5,59 meses). Dessas 94 crianças, 46 são do sexo feminino $(48,9 \%)$ e 48 do sexo masculino $(51,1 \%)$. A participação das crianças na pesquisa foi condicionada à assinatura, pelos responsáveis, do Termo de Consentimento Livre e Esclarecido. Essa pesquisa foi aprovada pelo Comitê de Ética em Pesquisa da Universidade Federal de Pernambuco (número de registro 064/08).

\section{Instrumentos}

Avaliação da Consciência Fonológica.

Tarefa de Subtração de Fonemas (tarefa desenvolvida tendo como base a tarefa de subtração de fonemas de Rosner \& Simon, 1971). Essa tarefa consiste na apresentação oral de uma palavra (p.ex.: /KaRta/) e o requerimento de que o participante, mentalmente, subtraia um som em particular (p.ex.: /K/) e diga o som que permaneceu (p.ex.: /aRta/). O som a ser subtraído se encontrava no início, no

${ }^{2}$ Os modelos 3 e 4 são compatíveis com a hipótese de Wolf e Bowers (1999), pois não importa para essa hipótese se a tarefa de memória fonológica é uma medida do processamento fonológico ou não, o que importa é que as tarefas de nomeação seriada rápida não são. meio ou no fim da palavra. $\mathrm{O}$ escore consistiu no número de itens respondidos corretamente. A tarefa contou com uma sessão de treinamento.

Tarefa de Spoonerismo (adaptada por Cardoso-Martins, Haase, \& Wood, 1998). A tarefa de spoonerismo requer que o participante troque o primeiro som de duas palavras apresentadas um pelo outro (Ex.: $/ \mathbf{m i L U}$ veRdi/ $=/$ vilLU meRdi/). A correção dessa tarefa se deu da seguinte forma, para cada item: a criança não recebeu pontos, quando errou as duas palavras; um ponto, quando acertou apenas uma palavra; e, dois pontos, quando respondeu corretamente as duas palavras. Essa tarefa também contou com uma sessão de treinamento.

\section{Avaliação da Nomeação Seriada Rápida (NSR).}

Tarefa de NSR de Letras (adaptada de Denckla \& Rudel, 1976). Foram selecionadas para compor essa tarefa as cinco letras mais frequentes para crianças da $3^{\mathrm{a}}$ série do ensino fundamental, tendo como base a lista de frequência de ocorrência de palavras de Pinheiro (1996). O participante era instruído a nomear, tão rápida e corretamente quanto possível, um conjunto de cinquenta estímulos visuais (correspondentes a 10 apresentações de cada uma das cinco letras, em ordem aleatória) dispostos em série em um cartão. Dois cartões foram utilizados e o escore de cada um consistiu no tempo gasto para se pronunciar o nome de todos os estímulos do cartão. O escore final foi a média do tempo gasto nos dois cartões. Antes de se iniciar a tarefa era verificado se o participante conhecia o nome dos estímulos.

Tarefa de NSR de Números (adaptada de Denckla \& Rudel, 1976). Os cinco números utilizados na tarefa de nomeação seriada rápida de números foram escolhidos entre os dez números de um único dígito pelo fato de serem todos dissílabos. Os procedimentos foram os mesmos da tarefa de nomeação seriada rápida de letras, porém considerando-se que os estímulos, nesse caso, eram números.

Tarefa de NSR de Objetos (adaptada de Denckla \& Rudel, 1976). Os cinco objetos utilizados na tarefa de nomeação seriada rápida de objetos são dissílabos. De uma forma geral, os objetos escolhidos para compor a tarefa de nomeação seriada rápida de objetos estão entre os cinco itens mais típicos de suas respectivas categorias, de acordo com os resultados do estudo de Pinheiro (2007) realizado com crianças brasileiras da $1^{\mathrm{a}}$ à $4^{\mathrm{a}}$ série do ensino fundamental. Os procedimentos foram os mesmos da tarefa de nomeação seriada rápida de letras, porém considerando-se que os estímulos, nesse caso, eram objetos.

Tarefa de NSR de Cores (adaptada de Denckla \& Rudel, 1976). As cores utilizadas na tarefa de nomeação seriada rápida de cores são as mesmas utilizadas por Denckla e Rudel (1976), pois as mesmas tiveram altos índices de tipicidade entre crianças brasileiras da $3^{\mathrm{a}}$ série do ensino fundamental (Pinheiro, 2007). Os procedimentos foram os mesmos da tarefa de nomeação seriada rápida de letras, porém considerando-se que os estímulos, nesse caso, foram cores. 
Avaliação da Memória de Trabalho Fonológica.

Subteste Dígitos da Escala de Inteligência Wechsler para Crianças-WISC-III (Wechsler, 2002). Esse subteste é formado por dois conjuntos: Ordem Direta e Ordem Inversa. Na Ordem Direta, a tarefa da criança consiste em repetir uma sequência de dígitos na mesma ordem enunciada pelo examinador. Na Ordem Inversa, a tarefa da criança consiste em repetir uma sequência de dígitos na ordem inversa daquela enunciada pelo examinador. $\mathrm{O}$ subteste foi administrado e corrigido de acordo com as especificações do manual (Wechsler, 2002).

\section{Procedimentos}

As crianças realizaram as tarefas na própria escola, em uma sala cedida pela coordenação. As tarefas foram administradas em sessões individuais, com duração de, aproximadamente, 15 minutos. O desempenho dos participantes em todas as tarefas administradas no presente es- tudo foi gravado. Esse procedimento permitiu a checagem posterior das informações anotadas nas folhas de resposta referentes a cada tarefa.

\section{Resultados}

A porcentagem de erros no desempenho nas tarefas de nomeação seriada rápida foi consideravelmente baixa (a tarefa de nomeação seriada rápida de cores foi a que apresentou a maior porcentagem de erros: $0,35 \%$ ) e, dessa forma, apenas o tempo médio levado para nomear os estímulos nas tarefas de nomeação seriada rápida foram considerados nas análises do presente trabalho.

As informações relativas ao escore máximo da tarefa, ao escore máximo obtido, ao escore mínimo obtido, à média, ao desvio padrão e à fidedignidade das tarefas administradas no presente estudo são apresentadas na Tabela 1.

Tabela 1

Estatísticas Descritivas e Fidedignidade das Tarefas Administradas

\begin{tabular}{lcccccc}
\hline Tarefas & Máx.T & Máx.O & Mín.O & Média & $D P$ & $r_{\mathrm{xx}}$ \\
\hline Spoonerismo & 20 & 20 & 0 & 14,83 & 4,88 & 0,88 \\
Subtração de Fonemas & 21 & 21 & 6 & 16,35 & 3,30 & 0,80 \\
Dígitos/WISC (escore ponderado) & 30 & 19 & 5 & 12,43 & 2,76 & 0,62 \\
NSR de Cores (seg.) & -- & 73,6 & 31,2 & 46,07 & 9,66 & 0,92 \\
NSR de Números (seg.) & -- & 49,61 & 18,5 & 28,61 & 5,81 & 0,92 \\
NSR de Objetos (seg.) & -- & 84,8 & 30,6 & 47,76 & 8,94 & 0,91 \\
NSR de Letras (seg.) & -- & 40,80 & 19,1 & 27,86 & 4,87 & 0,91 \\
\hline
\end{tabular}

Nota. NSR = Nomeação Seriada Rápida; Máx.T = Escore Máximo da Tarefa; Máx.O = Escore Máximo Obtido; Mín. $=$ Escore Mínimo Obtido; $D P=$ Desvio Padrão; $r_{\mathrm{xx}}=$ fidedignidade. A fidedignidade do teste 'Dígitos/WISC' foi obtida do manual do teste (Wechsler, 2002). Os valores de fidedignidade referentes às tarefas de nomeação seriada rápida representam o coeficiente de fidedignidade das duas metades, após a correção de Spearman-Brown (Hogan, 2006). Os valores referentes às demais tarefas são coeficientes alfa de Cronbach.

Como pode ser observado na Tabela 1 , todas as tarefas apresentaram índices de fidedignidade que variaram de aceitável $\left(r_{\mathrm{xx}}>0,60\right)$ a excelente $\left(r_{\mathrm{xx}}>0,90\right)$. Para avaliar se as variáveis tiveram uma distribuição normal, foram calculados, para cada variável, os escores das divisões do skewness pelo erro padrão do skewness e da kurtosis pelo erro padrão da kurtosis. Considerou-se como tendo uma distribuição normal todas as variáveis cujos escores resultantes dessas divisões foram maiores do que -1,96 e menores do que 1,96 (Dilalla \& Dollinger, 2006; Kline, 2005). Apenas a variável 'Dígitos/WISC' apresentou uma distribuição normal. As variáveis 'subtração de fonemas' e 'spoonerismo' apresentaram distribuições negativamente assimétricas e as variáveis 'nomeação seriada rápida de cores', 'nomeação seriada rápida de números', 'nomeação seriada rápida de objetos' e 'nomeação seriada rápida de letras' apresentaram distribuições positivamente assimétricas. Seguindo as recomendações de Garson (2009) e Kline (2005), essas variáveis foram submetidas às seguintes transformações logarítmicas: $\mathrm{L}_{\mathrm{n}}$ (valor máximo - escore +1) para as variáveis que apresentaram distribuições negativamente assimétricas e $\mathrm{L}_{\mathrm{n}}($ escore +1$)$ para as variáveis que apresentaram distribuições positivamente assimétricas. Após esses procedimentos, todas as variáveis transformadas apresentaram uma distribuição normal e, além disso, não houve nenhum outlier multivariado (o valor do coeficiente de Mardia ficou entre 1,96 e -1,96; Garson, 2009). 
Os modelos de mensuração derivados das propostas de Wagner e Torgesen (1987) e de Wolf e Bowers (1999) foram avaliados por meio de análises fatoriais confirmatórias desenvolvidas a partir do programa AMOS 7.0 (Analysis of Moment Structures). O método utilizado para o cálculo das estimativas foi o de Máxima Verossimilhança (Maximum Likelihood - ML). Um arquivo do programa SPSS for Windows versão 15.0 contendo os dados coletados foi utilizado como input.

Para a avaliação do ajuste dos modelos teóricos aos dados é comum os pesquisadores relatarem o resultado do teste qui-quadrado $\left(\chi^{2}\right)$ e o $p$ associado a ele. $\mathrm{O} \chi^{2}$ é um índice da significância da diferença entre a matriz de dados observada e a matriz estimada (modelo teórico proposto). Um bom modelo apresenta um $p$ associado ao valor de $\chi^{2}$ não significativo, indicando que o modelo teórico proposto não difere significativamente dos dados. Um problema desse índice é que ele é conhecido por ser sensível ao tamanho da amostra. Amostras grandes tendem a apresentar um $p$ significativo, enquanto amostras pequenas tendem a apresentar um $p$ não significativo. Outro índice que também é utilizado é o Índice de Qualidade do Ajuste (Goodness of Fit Index - GFI). Este índice é uma estimativa da proporção de variância explicada, com valores de GFI $>0,90$ indicando um bom ajuste do modelo aos dados e valores de GFI $<0,90$ indicando um ajuste pobre. De acordo com Kline (2005), o Índice de Ajuste Comparativo (Comparative Fit Index - CFI) e a Raiz Quadrada Média do Erro de Aproximação (Root Mean Square Error of Approximation - RMSEA) são os melhores índices do ajuste de um modelo aos dados por serem menos sensíveis ao tamanho da amostra. O CFI compara o modelo em teste com um modelo em que não há nenhuma covariação entre as variáveis. Os valores do CFI variam entre 0 e 1,0, sendo os valores acima de 0,95 indicativos de que o modelo proposto é um bom modelo. Por outro lado, o RMSEA é uma estimativa da quantidade de erros de aproximação do modelo dividido por seus graus de liberdade. Os valores do RMSEA variam entre 0 e 1,0, sendo os valores abaixo de 0,08 indicativos de um bom modelo. Assim sendo, para avaliar o ajuste dos modelos teóricos aos dados, esses quatro índices foram adotados no presente estudo.

Tabela 2

Índices de Ajuste dos Modelos aos Dados

\begin{tabular}{cccccccc}
\hline Modelos & $\chi^{2}$ & $p$ & $g l$ & GFI & CFI & RMSEA & RMSEA 90\% IC \\
\hline 1 & 57,6 & 0,001 & 12 & 0,87 & 0,84 & 0,202 & 0,152 a 0,256 \\
2 & 7,77 & 0,651 & 10 & 0,97 & 1 & 0,000 & 0,000 a 0,092 \\
3 & 7,77 & 0,651 & 10 & 0,97 & 1 & 0,000 & 0,000 a 0,092 \\
4 & 7,92 & 0,719 & 11 & 0,97 & 1 & 0,000 & 0,000 a 0,082 \\
\hline
\end{tabular}

Nota . Modelo 1 = modelo com um único fator; Modelo 2 = modelo com um fator de segunda ordem; Modelo $3=$ modelo com três fatores; Modelo 4 = modelo com dois fatores; GFI (Goodness of Fit Index) = Índice de Qualidade do Ajuste; CFI (Comparative Fit Index) = Índice de Ajuste Comparativo; RMSEA (Root Mean Square Error of Approximation) = Raiz Quadrada Média do Erro de Aproximação; IC = Intervalo de confiança.

A Tabela 2 apresenta os índices de ajuste de cada um dos modelos avaliados e a Figura 1 apresenta o diagrama relativo à avaliação do modelo de um único fator (Modelo 1$)^{3}$.

Como pode ser observado na Figura 1, os resultados relativos à avaliação desse primeiro modelo indicaram que, enquanto os caminhos ligando a variável latente 'processamento fonológico' a três das quatro medidas de nomeação seriada rápida (NSR de números, NSR de objetos e NSR de letras) não foram significativos ( $p>0,05)$, os caminhos ligando essa variável latente às medidas de subtração de fonemas, dígitos/WISC e NSR de cores foram significativos $(p<0,05)$. Considerando os índices que revelam o ajuste do modelo aos dados, o modelo teve um valor de $p$ associado ao qui-quadrado significativo, indicando que o modelo proposto e os dados observados foram significa- tivamente diferentes. Os demais índices, apresentados na Tabela 2, corroboram esse resultado.

Os resultados relativos à avaliação do modelo com três fatores de primeira ordem e um fator de segunda ordem (Modelo 2) podem ser encontrados na Figura 2.
${ }^{3}$ No presente estudo foi especificado que os erros de medida das variáveis 'NSR de cores' e 'NSR de objetos' seriam correlacionados, assim como os erros de medida das variáveis 'NSR de números' e 'NSR de letras'. Adotou-se esse procedimento porque há evidências de que dependendo da faixa etária e da escolaridade da amostra, itens não alfanuméricos (cores e objetos) e itens alfanuméricos (números e letras) são diferentemente impactados pela experiência, com a nomeação dos primeiros se tornando cada vez menos frequente e a nomeação dos últimos cada vez mais frequente (Wolf, 1991). 


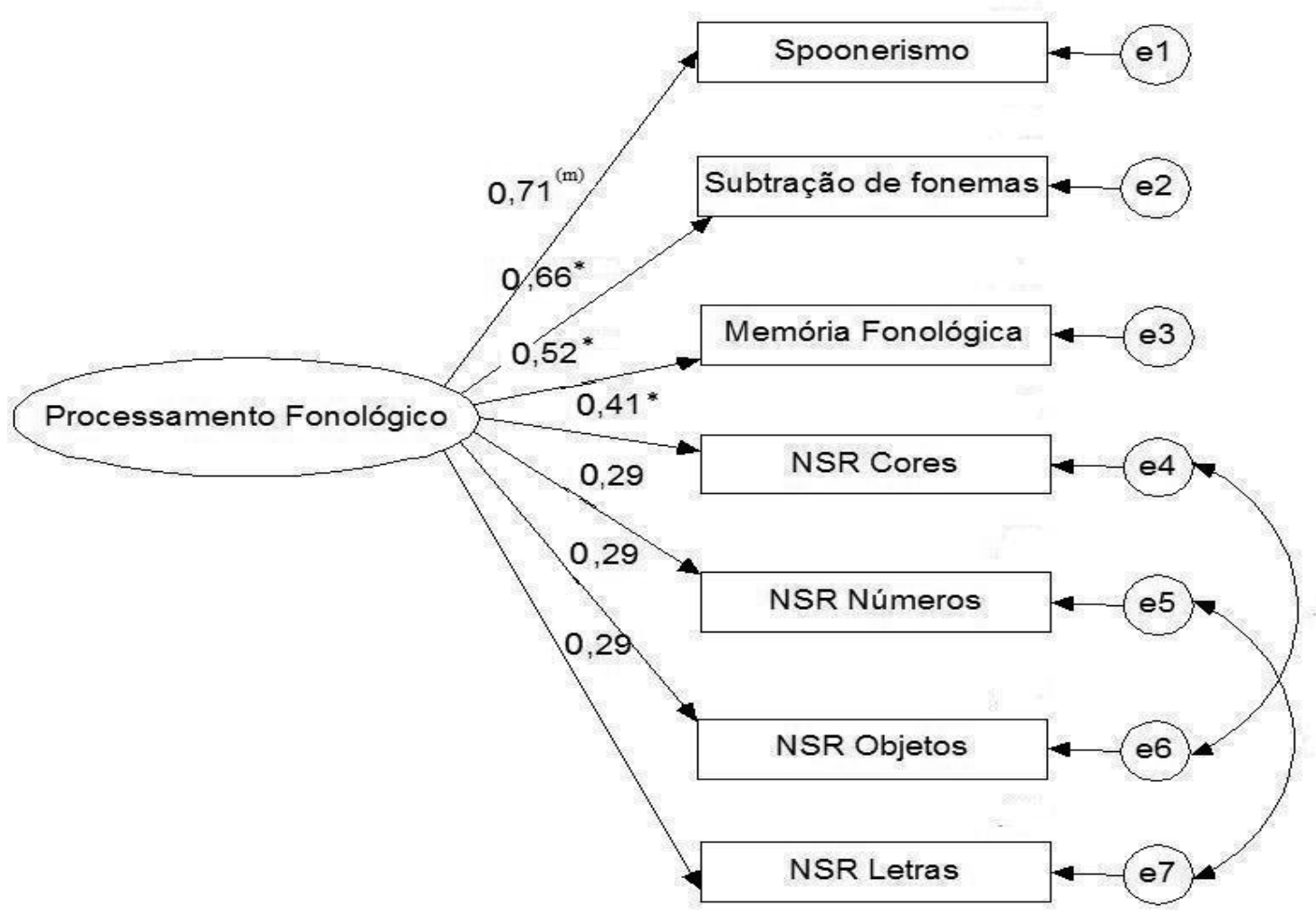

Figura 1. Diagrama do modelo com um único fator (representação da proposta de Wagner \& Torgesen, 1987). Nota . NSR $=$ nomeação seriada rápida $;(\mathrm{m})=$ métrica; $* p<0,05$.

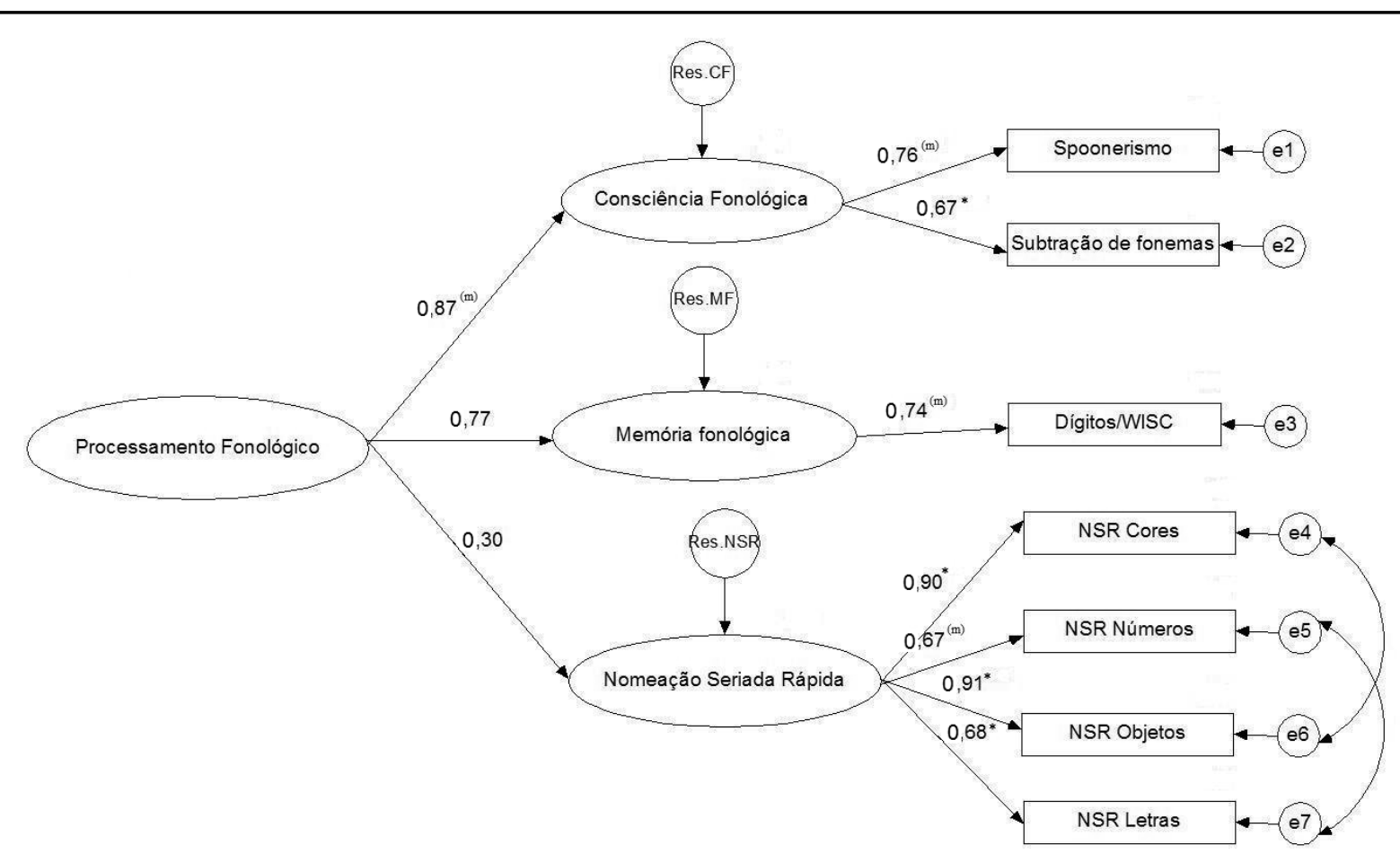

Figura 2. Diagrama do modelo com um fator de segunda ordem (representação da proposta de Wagner \& Torgesen, 1987). Nota $:$ NSR = nomeação seriada rápida; $(\mathrm{m})=$ métrica; $* p<0,05$. 
Como pode ser observado na Figura 2, os resultados relativos à avaliação desse segundo modelo indicaram que todos os caminhos que partem das variáveis latentes 'consciência fonológica', 'memória fonológica' ${ }^{\mathrm{e}}$ 'nomeação seriada rápida' até os seus respectivos indicadores foram significativos $(p<0,05)$. No entanto, os caminhos que partem do fator de segunda ordem - 'processamento fonológico' - para os fatores de primeira ordem - 'memória fonológica' e 'nomeação seriada rápida' - não foram significativos $(p>0,05)$. Como pode ser observado na Tabela 2, os índices que revelam o ajuste do modelo aos dados indicaram que esse modelo teórico é consistente com os dados.

$\mathrm{O}$ terceiro modelo avaliado pelo presente trabalho foi o modelo de três fatores (Modelo 3). A diferença em relação ao modelo 2 é que nesse modelo não há um fator de segunda ordem representando uma fonte comum de variação às variáveis latentes. Os resultados relativos à avaliação desse modelo podem ser encontrados na Figura 3.

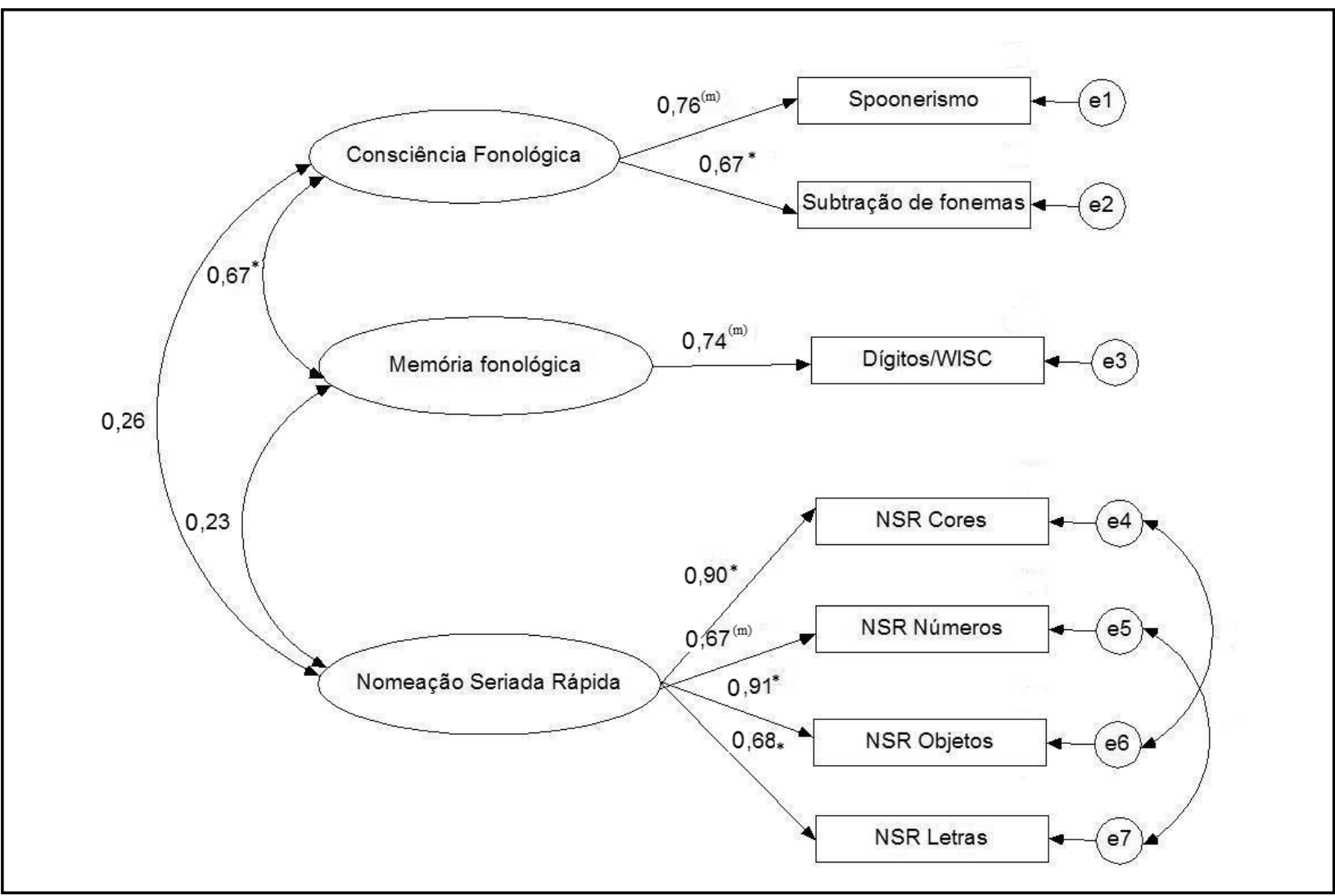

Figura 3. Diagrama do modelo com três fatores (representação da proposta de Wolf \& Bowers, 1999). Nota $:$ NSR $=$ nomeação seriada rápida $;(\mathrm{m})=$ métrica; $* p<0,05$.

Como pode ser observado na Figura 3, os resultados relativos à avaliação desse terceiro modelo evidenciaram
${ }^{4}$ Optou-se por tratar a variável 'memória fonológica' como uma variável latente (fator) porque, de um ponto de vista teórico, esse seria o procedimento mais justificado, uma vez que está se assumido que a tarefa 'digitos/WISC' é uma das formas de indexar a memória fonológica. Como, de acordo com Kline (2005), Coffman e MacCallum (2005) e Garson (2009), é possível ter uma variável latente com apenas um indicador, desde que a variância do erro de medida desse indicador possa ser fixada, adotou-se esse procedimento no presente estudo. Assim sendo, a variância do erro de medida da variável 'dígitos/WISC' foi calculada com base na fórmula [(D.P.) 2 x (1-rxx)]. Os valores referentes ao desvio padrão e à fidedignidade da medida foram retirados do manual do WISC (Wechsler, 2002). que todos os caminhos que partem das variáveis latentes até os seus respectivos indicadores foram significativos ( $p$ $<0,05)$. No entanto, no que diz respeito à correlação entre as variáveis latentes, apenas a 'consciência fonológica' e a 'memória fonológica' se correlacionaram significativamente. Os índices que revelam o ajuste do modelo aos dados indicaram que esse modelo teórico é consistente com os dados (vide Tabela 2).

O último modelo avaliado nesse estudo foi o modelo de dois fatores (Modelo 4). Os resultados relativos à avaliação desse modelo podem ser encontrados na Figura 4.

Como pode ser verificado na Figura 4, os resultados da avaliação desse último modelo indicaram que todos os caminhos que partem das variáveis latentes aos seus respectivos indicadores foram significativos $(p<0,05)$. Além 
disso, os resultados evidenciaram que as variáveis latentes 'processamento fonológico' e 'nomeação seriada rápida' não se correlacionaram significativamente $(r=0,27, p>$ $0,10)$. Como pode ser observado na Tabela 2 , os índices que revelam o ajuste do modelo aos dados indicaram que esse modelo teórico é consistente com os dados.

Uma vez que dois ou mais modelos se mostram consistentes com os dados, é importante observar alguns critérios para a escolha de um modelo em detrimento dos outros. Kline (2005), comentando a esse respeito, afirma que tanto critérios estatísticos como teóricos devem ser considerados. Do ponto de vista estatístico, um índice que leva em consideração o grau de parcimônia dos modelos e, assim sendo, permite a comparação entre eles é o Consistent Akaike Information Criterion (CAIC). Esse índice dá informações sobre o grau de parcimônia do modelo por meio de uma relação entre o número de parâmetros estimados e o número de pontos de dados na matriz de covariâncias. O modelo que obtiver o menor valor de CAIC é o mais parcimonioso (Kline, 2005). Destarte, considerando-se o índice CAIC, pode-se dizer que o 'Modelo 4' (modelo de dois fatores) foi o mais parcimonioso, pois apresentou um CAIC de 102,18, enquanto o 'Modelo 3' e o 'Modelo 2' apresentaram índices CAIC maiores que 107,00. Como o 'Modelo 1' não foi consistente com os dados, o CAIC relativo a esse modelo não foi considerado. Questões teóricas serão apresentadas na seção 'discussão'.

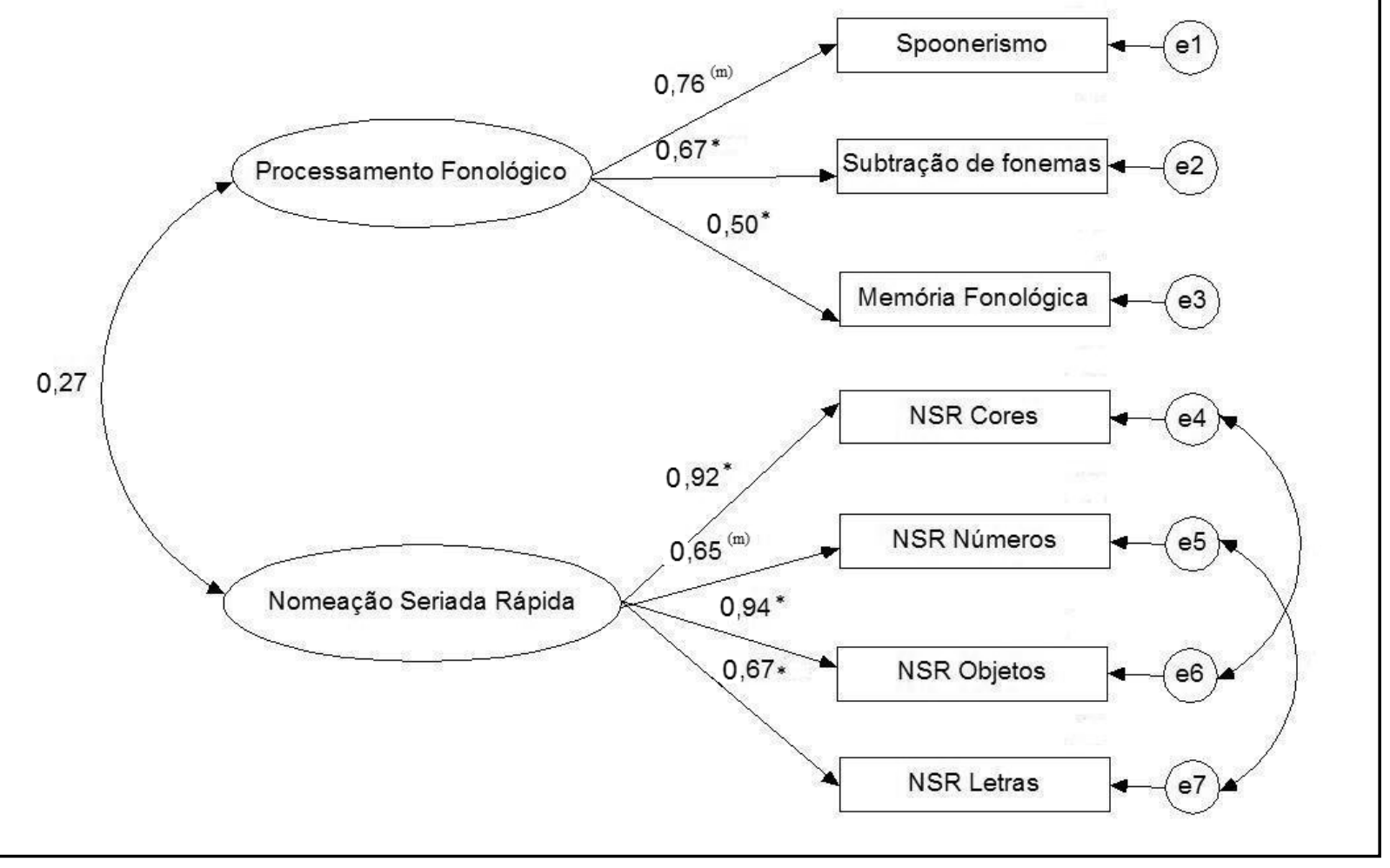

Figura 4. Diagrama do modelo com dois fatores (representação da proposta de Wolf \& Bowers, 1999).

Nota $:$ NSR $=$ nomeação seriada rápida; $(\mathrm{m})=$ métrica; $* p<0,05$.

\section{Discussão}

Os resultados do presente estudo não dão suporte à proposta de Wagner e Torgesen (1987), uma vez que o modelo de um único fator (Modelo 1) não foi consistente com os dados e no modelo com um fator de segunda ordem (Modelo 2) os caminhos que ligam a variável de segunda ordem 'processamento fonológico' às variáveis 'memória fonológica' e 'nomeação seriada rápida' não foram significativos, indicando que esses caminhos poderiam ser removidos do modelo. Esse modelo foi consistente com os dados porque associado a cada fator de primeira ordem está um 'resíduo', representando todas as causas de variações nesses fatores não provenientes do fator de segunda ordem (causas extrafonológicas, no caso). Dessa forma, mesmo não sendo significativo o caminho que liga o fator de segunda ordem aos fatores de primeira ordem, está contemplada no modelo outra fonte de variações, o que torna o modelo consistente com os dados mesmo quando os caminhos não são significativos (Kline, 2005). É importante ressaltar, no entanto, que o fato dos caminhos que ligam o fator de segunda ordem aos de primeira ordem não terem sido significativos é de relevância considerável para a questão investigada no presente trabalho. Afinal, que 
sentido teria aplicar tarefas de nomeação seriada rápida para avaliar o processamento fonológico se, conforme o resultado da avaliação do segundo modelo, o processamento fonológico não foi responsável por uma variação significativa na nomeação seriada rápida? Assim sendo, nem a análise do modelo de um único fator, nem a análise do modelo com um fator de segunda ordem apresentaram resultados que corroboram a hipótese de Wagner e Torgesen (1987).

É importante ressaltar que em um estudo recentemente publicado, Nelson, Lindstrom, Lindstrom e Denis (2012) avaliaram a adequação de um modelo com uma estrutura fatorial idêntica ao modelo 2 do presente estudo em falantes da língua inglesa. Apesar de Nelson et al. não relatarem a significância dos caminhos que ligam o fator de segunda ordem aos fatores de primeira ordem, eles relataram que o fator de primeira ordem 'nomeação seriada rápida' apresentou uma variância residual de $65 \%$, indicando que, assim como no presente estudo, ele não foi fortemente dirigido pelo fator de segunda ordem 'processamento fonológico'. Esse resultado é interessante, pois indica que mesmo em línguas com uma consistência grafema-fonema diferente, como as línguas inglesa e portuguesa, a nomeação seriada rápida não parece ser melhor categorizada como uma medida do processamento fonológico.

Além de não oferecer suporte para a proposta de Wagner e Torgensen (1987), os resultados do presente estudo parecem corroborar a proposta de Wolf e Bowers (1999), já que tanto o Modelo 3 como o Modelo 4 foram consistentes com os dados e em ambos o fator 'nomeação seriada rápida' não se correlacionou significativamente com os demais fatores. Ademais, é importante considerar, também, que o Modelo 4 foi o mais parcimonioso de todos, devendo ser considerado, portanto, dentre os modelos avaliados no presente trabalho, o melhor modelo da relação existente entre as medidas de consciência fonológica, memória de trabalho fonológica e nomeação seriada rápida e os construtos subjacentes a essas medidas.

Uma questão que merece ser considerada, novamente, é o argumento de Wagner e Torgesen (1987) e Wagner et al. (1993) de que o modelo de três fatores é compatível com a hipótese teórica de que as tarefas de nomeação seriada rápida avaliam o processamento fonológico, pois, segundo eles, essas tarefas avaliam um tipo de processamento fonológico diferente do avaliado pelas tarefas de consciência fonológica e memória de trabalho fonológica e, por isso, é compreensível que essas tarefas sejam indicadoras de um fator separado dos demais fatores. Esse argumento parece ser equivocado, porque o modelo que parece melhor representar essa ideia é o modelo que inclui um fator de segunda ordem (processamento fonológico) ligado aos três fatores de primeira ordem (consciência fonológica, memória fonológica e nomeação seriada rápida) e não o modelo de três fatores. Apenas o modelo com um fator de segunda ordem, de fato, representa a ideia de que as tarefas de consciência fonológica, de memória fonológica e nomeação seriada rápida avaliam três tipos de processa- mento fonológico diferentes, posto que, o modelo de três fatores não prediz uma fonte de variações que seja comum a todos os fatores (por exemplo, o que leva a consciência fonológica se correlacionar com a nomeação seriada rápida não necessariamente é o que leva a memória fonológica se correlacionar com a nomeação seriada rápida). Independente disso, é importante considerar que mesmo no modelo de três fatores as correlações entre a nomeação seriada rápida e as demais variáveis foram fracas e nem chegaram a ser significativas.

\section{Conclusão}

Com base no presente estudo, pode-se dizer que a melhor forma de se conceber a relação entre os escores nas tarefas de consciência fonológica, de memória de trabalho fonológica e de nomeação seriada rápida e os construtos subjacentes a eles é através de um modelo de dois fatores no qual as medidas de nomeação seriada rápida representam um fator separado do fator 'processamento fonológico'. No entanto, apesar dos resultados desse estudo corroborarem a hipótese de Wolf e Bowers (1999) e colocarem em dificuldades a hipótese de Wagner e Torgesen (1987), é importante considerar que a natureza exata dos processos subjacentes à nomeação seriada rápida não foi investigada. Esse estudo apenas traz evidências de que a natureza desses processos não é, primordialmente, fonológica (o que é importante porque torna injustificável a inclusão dessas tarefas em baterias de testes que visam à avaliação do processamento fonológico). Dessa forma, é imprescindível que estudos futuros investiguem com maior especificidade a natureza dos processos subjacentes à nomeação seriada rápida, tendo em vista o grande número de estudos que evidenciam a contribuição dessa variável para o desenvolvimento da leitura. Compreender melhor a natureza dessa tarefa será um passo importante para a realização de um diagnóstico adequado de dificuldades de leitura, bem como é um passo indispensável para a elaboração de um programa de intervenção que tenha como objetivo estimular os processos subjacentes à nomeação seriada rápida, visando o desenvolvimento da leitura.

\section{Referências}

Anthony, J., Williams, J., McDonald, R., \& Francis, D. (2007). Phonological processing and emergent literacy in younger and older preschool children. Annals of Dyslexia, 57, 113-137.

Baddeley, A. (2000). Short term and working memory. In E. Tulving \& F. Craik (Eds.), The Oxford handbook of memory (pp. 77-92). New York: Oxford University Press.

Bryant, P., \& Bradley, L. (1987). Problemas de leitura na criança. Porto Alegre, RS: Artes Médicas.

Cardoso-Martins, C., Haase, V., \& Wood, G. (1998). Bateria de testes de habilidades fonológicas adaptada da Phonological Assessment Battery. Manuscrito não publicado.

Coffman, D., \& MacCallum, R. (2005). Using parcels to convert path analysis models into latent variable models. Multivariate Behavioral Research, 40, 235-259. 
de Jong, P., \& van der Leij, A. (1999). Specific contributions of phonological abilities to early reading acquisition: Results from a Dutch latent variable longitudinal study. Journal of Educational Psychology, 91, 450-476.

Denckla, M., \& Rudel, R. (1976). Rapid automatized naming (RAN): Dyslexia differentiated from other learning disabilities. Neuropsychology, 14, 471-479.

Dilalla, D., \& Dollinger, D. (2006). Cleaning up data and running preliminary analysis. In F. Leong \& J. Austin (Eds.), The psychology research handbook: A guide for graduate students and research assistants (pp. 167-176). London: Sage.

Garson, D. (2009). Structural equation modeling. Retrieved August 22, 2009, from http:/faculty.chass.ncsu.edu/garson/ PA765/structur.htm

Hogan, T. (2006). Introdução à prática de testes psicológicos. Rio de Janeiro, RJ: LTC.

Kline, R. (2005). Principles and practice of structural equation modeling. London: The Guilford Press.

Lonigan, C., Anthony, J., Phillips, B., Purpura, D., Wilson, S., \& McQueen, J. (2009). The nature of preschool phonological processing abilities and their relations to vocabulary, general cognitive abilities, and print knowledge. Journal of Educational Psychology, 101, 345-358.

Lovett, M., Steinbach, K., \& Frijters, J. (2000). Remediating the core deficits of developmental reading disability: A double-deficit perspective. Journal of Learning Disabilities, 33, 334-358

Nelson, J., Lindstrom, J., Lindstrom, W., \& Denis, D. (2012). The structure of phonological processing and its relationship to basic reading. Exceptionality: A Special Education Journal, 20, 179-196

Norton, E., \& Wolf, M. (2012). Rapid Automatized Naming (RAN) and reading fluency: Implications for understanding and treatment of reading disabilities. Annual Review of Psychology, 63, 427-452.

Pinheiro, A. (1996). Contagem de frequência de ocorrência e análise psicolinguística de palavras expostas a crianças na faixa pré-escolar e séries iniciais do $1^{\circ}$ grau. São Paulo, SP: Associação Brasileira de Dislexia.

Pinheiro, A. (2007). Levantamento dos membros de categorias semânticas para itens verbais produzidos de forma escrita por crianças [Resumo]. In V. Golveia, C. Hutz, W. Lourenzo, \& E. Almeida (Eds.), III Congresso Brasileiro de Avaliação Psicológica e XII Conferência Internacional de Avaliação Psicológica: Formas e contextos - Avaliação psicológica no século XXI: Ética e ciência - Livro de resumos/mesas redondas (pp. 34-35). João Pessoa, PB: Instituto Brasileiro de Advocacia Pública.

Powell, D., Stainthorp, R., Stuart, M., Garwood, H., \& Quinlan, P. (2007). An experimental comparison between rival theories of rapid automatized naming performance and its relationship to reading. Journal of Experimental Child Psychology, 98, 46-68.

Rosner, J., \& Simon, D. (1971). The auditory analysis test: An initial report. Journal of Learning Disabilities, 4, 384-392.

Taibah, N., \& Haynes, C. (2011). Contributions of phonological processing skills to reading skills in Arabic speaking children. Reading and Writing, 24, 1019-1042.

Torgesen, J., Wagner, R., Rashotte, C., Burgess, S., \& Hecht, S. (1997). Contributions of phonological awareness and rapid automatic naming ability to the growth of word-reading skills in second - to fifth - grade children. Scientific Studies of Reading, 1, 161-185.
Vandewalle, E., Boets, B., Ghesquière, P., \& Zink, I. (2012). Development of phonological processing skills in children with Specific Language Impairment with and without literacy delay: A 3-year longitudinal study. Journal of Speech, Language, and Hearing Research, 55, 1053-1067.

Wagner, R., \& Torgesen, J. (1987). The nature of phonological processing and its causal role in the acquisition of reading skills. Psychological Bulletin, 101, 192-212.

Wagner, R., Balthazor, M., Hurley, S., Morgan, S., Rashotte, C., Shaner, R., ...Stage, S. (1987). The nature of prereaders' phonological processing abilities. Cognitive Development, 2, 355-373.

Wagner, R., Torgesen, J., \& Rashotte, C. (1994). Development of reading-related phonological processing abilities: New evidence of bidirectional causality from a latent variable longitudinal study. Development Psychology, 30, 73-87.

Wagner, R., Torgesen, J., Laughon, P., Simmons, K., \& Rashotte, C. (1993). Development of young readers' phonological processing abilities. Journal of Educational Psychology, 85, 83-103.

Wagner, R., Torgesen, J., Rashotte, C., Hecht, S., Barker, T., Burgess, S., ...Garon, T. (1997). Changing relations between phonological processing abilities and word-level reading as children develop from beginning to skilled readers: A 5-year longitudinal study. Developmental Psychology, 33, 468-479.

Wechsler, D. (2002). WISC-III: Escala de inteligência Wechsler para crianças. Manual (3. ed.). São Paulo, SP: Casa do Psicólogo.

Wolf, M. (1991). Naming speed and reading: The contribution of the cognitive neurosciences. Reading Research Quarterly, 26, 123-141.

Wolf, M., \& Bowers, P. (1999). The double-deficit hypothesis for the developmental dyslexias. Journal of Educational Psychology, 91, 415-438. 